\title{
Augmented Reality
}

\author{
Shazab Rehman Shakeel \\ Department of CS \& IT, \\ University of management \& Technology University \\ Usman Shahbaz \\ Department of CS \& IT, \\ University of management \& Technology University \\ Danish Shafeq Khan \\ Department of CS \& IT, \\ University of management \& Technology University
}

\begin{abstract}
In this paper describe the general ideal feature of Augmented Reality (AR) plus central conception of this type of technology. At this time it is also describe the major ground of $A R$ and is also useful in that particular ground and tells about the very important AR devices. Future guidelines are also discussed and AR depended future technologies explain. A few uniqueness of Augmented Reality systems and devices will be talk about and this paper also will explain about AR overview.
\end{abstract}

Keywords: Augmented Reality, Virtual Reality, Scientific Visualization

\section{INTRODUCTION}

Technology of AR that includes the graphics of computer and wraps it on the actual world. Best overviews about technology are (R. Azuma, 1995) that distinct the fields express more troubles and add all developed point. With the intention of paper gives an exciting preliminary point for everyone in researching or using segmented reality.

Most common term for AR is mixed reality (MR) (P. Milgram et al., 1993), which mention the domain that cover up telepresence, AR, VR and further technologies.

Virtual reality is 3D environment that produce by computer that allow user use, go into and cooperate with artificial atmosphere (A. Van Dam et al., 2003) (W. Sherman et al., 2003) (R. Silva et al., 2003). User is capable to "submerge" them to unstable level in the computers nonnatural world which might moreover be an imitation of a little shape of certainty (P. du Pont, 1995) or complex phenomenon imitation (D. Weimer, 1994) (A. Van Dam et al., 2000).

In the telepresence, the main reason is to expand facilities of operators of sensory-motor and problem solving capability to an atmosphere of remote.

logically, telepresence be able to describe as a human/machine system in which the human being operative get adequate information regarding the telephone operator and mission situation, present into a adequately usual way, that the operative sense actually in attendance at the isolated site (T.B. Sheridan, 1992). Extremely comparable to virtual reality, within which we decide to attain the delusion of existence inside a computer reproduction, telepresence aim to get the delusion of existence at an isolated location. 
AR is like comparing a technology among virtual reality and telepresence. even as in VR the While in telepresence it's totally real, in VR the whole environment is totally artificial and in AR the user experience the actual world augmented and that have a virtual objects. During design an AR system, kept three important phases in mind:

- Mixture of virtual and real world

- Capable of acting on real time

- Entry in 3d

Wearable devices, like Head-Mounted-Displays (HMD), are use to prove augmented sight/picture, but there are also further similar technologies are accessible (R. Azuma, 1997).

Moreover talk about three phases, one more might be integrated: Portability. Approximately in the entire of the virtual environment systems, the user can't go about a lot because of limitation devices. Though, various AR applications will require that user in fact walk from side to end a large environment. Therefore, portability turns out to be an important issue.

For such kind of application, the registration of 3D become more complex, body-borne computers application usually provide unregistered, using monocular HMD for text or graphics.

These are like "see-around" system and not like AR system by the thin explanation. From now on computer plate forms and body-borne exhibit devices used by AR have to be Repeatedly developed for further common applications

Over one decade augmented reality field has been present and in the few past years the progress in this field is amazing. (R. Azuma et al., 2001). Since (R. Azuma, 1997), the field has developed quickly. Quite a lot of meeting dedicated in this domain were started, together with the intercontinental workshops and seminar on Augmented Reality, the worldwide conference taking place varied Reality as well as AR designing environment workshops.

\section{AR Devices}

\section{LITERATURE REVIEW}

There are four main classes of AR in display type: See-Through Optical, Virtual Retinal Systems, See the video Through HMD, Monitor using AR and AR on project. The following sections give you an idea about the equivalent devices and show their key features.

\section{See-Through Optical HMD}

In A See-Through optical AR makes use of visible Head Mounted Display toward show the effective atmosphere straight above the actual planet.

Because of insertion optical combiners it works like it happen in the front of eyes of user. The combiner is divided, because of that the user can see straight genuine globe. Combiner reflects also, as results the user observe virtually image spring back the combiners as of HMD. Examples of major See-Through Optical are a variety of medical systems augmented.

\section{Virtual Retinal Systems}

In 1991, at Washington University Virtual Retinal Display was developed in the Human Interface Technology Lab. Main objective is to build a colorful, broad field with view, vast brightness, and huge resolution, virtually display with low price. Micro visions Inc. have commercialized VRD technology because of exclusive license. 
This technology provides lots of possible application, like head-mounted displays Used for military and also for aerospace application for medical reason.

\section{See the video through HMD}

See the video through HMD technology uses a thick HMD to show combined video of the VE and view through camera lying on the HMD. This come within reach of is little difficult than AR in see-through optical, have need of appropriate position to camera .On the other hand, composition of video of the real and virtual worlds is a lot easier.

\section{Monitor Using AR}

AR Monitor technology use combined film flow other than the display is similar to desktop monitor. Possibly there is s slightest hard setup of AR, while it gets rid of HMD matter. Princeton Image of video, Inc. have created a method for integration picture and put it in real time video streams. We see this work in American foot ball games and also used for broadcasts the advertising logos.

\section{Projection Displays}

Because of virtual environment AR technology of projection used which project the object of real world. AR projection as well sounds fit for numerous user circumstances. Arrangement and surface of projection is serious used for unbeaten applications. The application is used in it is like engineering assembly, manufactured goods vision, etc.

\section{Applications}

Augmented Reality expertise has countless potential applications in a broad variety of domains; consist of education, engineering, entertainment, medicine and developed. Anticipated with the aim of further possible fields of applications will show through broadcasting technology.

\section{Medical}

In medical domain using of imaging technology is mostly advanced, not of astonishing to medical area will investigation other essential used for AR systems. There are majority applications of medical field which is work with the surgery guide by image.

An additional application of AR in medical field is like ultrasound of imaging. (S. Andrei et al., 1993) simply use of optical see-through to see by the ultrasound skilled worker and through this technology you can also view and examined in the abdomen of women which is pregnant. This image show within of the abdomen and perfectly identify the problems if have in it. (R. Azuma, 1993)

\section{Entertainment}

Simple kind of augmented reality is used for entertainment, news and also for business purposive. Every time when the individual see the report of weather in evening, the reporter in only stand in the front of shifting maps of weather. If we talk about studio same technique in used like the reporter is stand in the front of blue screen. One more entertainment domain is game development in AR (Z. Szalavri et al., 1998).Actual image in augmented reality is computer generate it's by use a technology called Chroma-keying.

\section{Military Training}

Militarily mostly using AR in a kind of area that shows information to the pilot on the screen of the flight helmet. Also a kind of display in augmented reality. 


\section{Robotics and Tele robotics}

Augmented display is able to help the user in the domain of robotics and also in tele-robotics. (W.S. Kim et al., 1993)(P. Milgram et al., 1993). Tele-robotics operator is using visual image inside remote space of work to provide guideline to the robot. The view might be helpful when the operator see the scene in front of him. Moreover, the view might make the visualization easy for the $3 \mathrm{~d}$ remote of geometry.

\section{Manufacturing, Maintenance and Repair}

After the maintenance of technician advance unknown part of tools as an alternative of opening quite a lot of repair manuals and they might place on AR display. The display of the image is correlate with augmented that is innovative and related information has to be repair. E.g., attachment of hardware and position of fasteners that have to be removed which is highlighted.

\section{Visualization Issues}

There are Researchers that have starting troubles in showing information inside AR, Studiers tube collaborative AR structure shows the environment of AR expertise. All the work is done to correct the registration mistakes or error and stay away from sensitive data because of core problems.

\section{Visualization Errors}

For many AR system, mistakes are major and can't avoidable. E.g., the position of object Inside environment might be not recognized correctly sufficient to stay away from noticeable registration mistake. Below such circumstances, one move toward for representation visual display of object and his domain of field wherever the object possibly will exist in, it is also base on following and capacity mistakes (B. Macintyre et al., 2000).these gives warranty that virtual illustration forever holds the real matching part.

\section{Removing real objects from the environment}

To remove real objects are further beside out depth information from a sight. The systems have to as well be capable to division being objects in that environment Auto loading technique, to verify objects and their position (V. Lepetit et al., 2000). These make possible the addition of virtual objects and removal of actual objects with no a clear 3D renewal of environment.

\section{Photorealistic Rendering}

To upgrade the service quality of virtual object a key is needed in AR application automatically has ability to catch the environmental lighting information (S. Boivin et al., 2001) (Y. Mukaigawa et al., 1999) (J. Stauder, 1999).

e.g.,(D. Cobzas et al., 2003) there is a technique, make a use of camera, permit to catch the object of configuration and manifestation, after that, later on stage, rendering and AR cover into a fresh sight.

\section{AR in Event Planning}

\section{AUGMENTED REALITY SOLVING REAL LIFE PROBLEMS}

\section{AR Cube}

AR Cube is like a small or mini-information portal. It is on the whole an interactive cube that serves as single go-to storage for all the key event information. Fist AR Cub was applied as component of Global Innovation Forum to offer participants a sci-fi know-how of exploring conference content, such as: 
- Conference schedule

- Information about speakers

- Information about sponsors

- Other useful info about conference

Another worthy cause is behind of Cube designing - it helps by save the environment by dumping paper waste: one dynamic information portal as replacement of ton of printed media, such as fliers and catalogs, booklets. In addition, positioning event controller as smarter and extremely innovative, AR Cube also can helps share and built an information with easy way.

\section{AR in E-Commerce}

Mobile e-commerce keeps growing at a huge speed. In 2019, an expected 1.92 billion people around the world will buy products online where approximately $67 \%$ of all purchase is made on a mobile device. Therefore it is so necessary to make sure an excellent shopping experience for mobile users.

\section{Virtual Try-on of Watches}

When people see a product they like what they do? They want to use it. What the people do when they see a product on internet which is desirable for them? They surprise if it will work for them. If that particular product is a watch, you have got it covered

A design rush certified startup called AR-Watches allows users to try on watches while shopping online virtually.AR- Watched is essentially an app developed for both Android and IOSs, which let users to try on watches from virtually different brands.

It comes up to with a paper wristband, which dish up as a marker for AR to place on top the 3D image of watches onto a user's wrist.

\section{This solution has 3 main benefits:}

1) It can help to cut down on returns considerably.

2) It can make a bridge among physical and online stores when the marker is given to customers to take home and play around with different styles.

3) It can be used for safety purposes, when watches are tried on virtually rather than taking each item out of shelf and increasing risk of damage.

\section{AR Menu: A Virtual Preview of Food Orders}

Another app called Jar it (meaning fried potatoes!) has opened in recent times to allow people to see their possible food order in 3D before in fact placing an order.

The AR food preview experience can be used not only for online food ordering, but also for restaurants and food chains.

This solution's main benefit is that it enables customers to make informed decisions on their order, which, in its turn, reduces the chances of a customer not liking the dish's appearance.

\section{AR in Political Campaigns}

One very uncommon, yet efficient use of AR is in political campaigns, supporting social causes. If scanned with the Arloopa app, the person in the image below will come to life with an important message. 
The person is Minister of Education and Science of Armenia A. Harutyunyan, and this is his election campaign poster, which by itself was a clear, straightforward message to people that they were going to have an innovation-oriented education leader.

\section{AR in Education}

Interactive Books

Traditional methods of education are gradually becoming a thing of the past, making way for innovations.

\section{Among the most significant trends in education technology, augmented reality rightfully takes a leading position.}

AR has a skill to deliver objects that are tough to imagine and show them in the form of 3D models, hence making it easier for students to grab the abstract and difficult content. Interactive textbooks also increase students' engagement and make the learning process a lot more fun and rewarding.

\section{Distance learning}

AR-aided materials help students learn even outside the classroom. Add to that fact, online or distance learning can be easier and more fun with interactive AR content.

For instance, Mondly, a language-learning app, has integrated an AR-powered virtual tutor to help users practice their skills as if they were in a real-life setting.

\section{AR in Interior Design}

Architects and designers are slowly put together AR experiences in their business, as do online furniture stores. AR helps in the endorsement of their new decoration ideas, giving a realistic picture of items, making customers experience confident before they buy.

In home decoration a great usage of $\mathrm{AR}$ is by interiAR, a startup that features an interactive $\mathrm{AR}$ app (Android/OS). The app allows users to decorate their own room in real time by simply dragging and dropping furniture items into their home.

\section{RESEARCH METHODOLOGY}

In this research study using the wide use if both secondary and primary source of data. The research includes the study of augmented reality and its key factors. This research include the process of study different factors that affect AR, environment of market, landscape of competitive, historical data, current using technology in market, innovative technologies, future technologies and technical progress in related industry and market risks, opportunity, market obstacle and challenges.

\section{OBJECTIVES}

The objective of this paper is to identify the potential of AR and its application in real life that are available in different domains. What types of AR applications are currently being used?

Another objective about Augmented Reality is to create a system in which the user cannot tell the difference between the real world and the virtual augmentation of it Today Augmented Reality is used in entertainment, military training, engineering design, robotics, manufacturing and other industries. 


\section{CONCLUSION}

There is a lot of advancement in AR but most of work leftovers need to be done. By using available libraries can help to developed Application. ART toolkit is one of them, (M. Billinghurst et al., 1997) because of computer vision technique that give to compute position of camera and direction to marked the comparatively cards consequently that virtual 3D objectives accurately on the markers.

If AR is to become normally set up then here are some regions need further research.

Ever-present tracking and system portability: a number of remarkable AR expressions have produce convincing atmosphere with closely pixel-accurate registration. On the other hand, such expressions work just indoors limited, watchfully arranged surroundings.

The vital objective is a supports ideal registration of tracking system in any random unsuspecting environment, inside or outside. Permitting AR systems wherever to go which as well need that are at plus inconspicuous.

Use and ease of system: Expert user are necessary for AR systems (in general the System maker) to regulate as well as work with them. If AR application are turn out to be an ordinary place, the system must be regulate and also operate by user with non experience. This demand more strong systems so as to keep away from or reduce make fine adjustments and requirements of setup.

Photorealism and advanced depiction: even although lots of AR applications just require simple graphics like wireframe outlines and text labels, the vital aim is to turn into the virtual objects distinct from the genuine. Into actual time this has to finish, not including the manual involvement of programmers. Image based fresh technologies interpretations have to be measured in organize for complete job (S. Boivin et al., 2001).

Entire augmented reality senses: paying attention by researchers mainly on augment the illustration sense. Finally, convincing AR situation might need conn ect further senses as well (hearing, touching, etc.)

\section{References}

K. Ahlers and A. Kramer. Distributed augmented reality for collaborative design applications. European Computer Industry Research Center, 3-14, 1995.

S. Andrei, D. Chen, C. Tector, A. Brandt, H. Chen, R. Ohbuchi, M. Bajura, and H. Fuchs. Case study: Observing a volume rendered fetus within a pregnant patient. Proceedings of IEEE Visualization, 17-21, 1993.

R. Azuma. Tracking requirements for augmented reality. Communications of the ACM, 36(7):50-51, 1993.

R. Azuma. A survey of augmented reality.ACM SIGGRAPH, 1-38, 1997.

M. Billinghurst, S. Baldis, E. Miller, and S. Weghorst. Shared space: Collaborative information spaces. Proc. of HCI International, 7-10, 1997.

M. Billinghurst and H. Kato. Mixed reality - merging real and virtual worlds. Proc. International Symposium on Mixed Reality (ISMR'99), 261-284, 1999.

S. Boivin and A. Gagalowicz. Imagebasedrendering for industrial applications.ERCIM News, 2001.

D. Cobzas, K. Yerex, and M. Jagersand. Editing real world scenes: Augmented reality with image-based rendering. Proc. of IEEE Virtual Reality, 291-292, 2003.

A. Van Dam, A. Forsberg, D. Laidlaw, J. LaViola, and R. Simpson. Immersive VR for scientific visualization: A progress report. IEEE Computer Graphics and Applications, 20(6): 26- 52, 2000. 
P. du Pont. Building complex virtual worlds without programming. EUROGRAPHICS' 95 State Of The Art Reports, 61-70, 1995.

A. Fuhrmann et. al. Occlusion in collaborative augmented environments. Computers Graphics, 23 (6): 809-819, 1999.

R. Azuma et al. Recent advances in augmented reality. IEEE Computer Graphics and Applications, 20-38, 2001.

R. Chinthammit et al. Head tracking using the virtual retinal display. Second IEEE and ACM International Symposium on Augmented Reality, 235-242, 2001.

Z. Szalavri et. al. Studierstube: An environment for collaboration in augmented reality. Virtual Reality Systems, Development and Application, 3 (1): 37-48, 1998.

W. Grimson, G. Ettinger, T. Kapur, M. Leventon, W. Wells, and R. Kikinis. Utilizing segmented MRI data in imageguided surgery. International Journal of Pattern Recognition and Artificial Intelligence, 11(8):1367-97, 1998.

Richard Lee Holloway. Registration errors in augmented reality systems. Technical Report TR95-016, the Universityof North Carolina, 11995.

W. S. Kim and P. Schenker. An advanced operator interface design with preview/predictive displays for groundcontrolled space telerobotic servicing. Proceedings of SPIE Vol. 2057: Telemanipulator Technology and Space Telerobotics, 96-107, 1993.

V. Lepetit and M. Berger. Handling occlusions in augmented reality systems: A semi-automatic method. Proc. Int.l Symp. Augmented Reality, 137-146, 2000.

B. MacIntyre and E. Coelho. Adapting to dynamic registration errors using level of error (loe) filtering. Proc. Int.l Symp. Augmented Reality, 85-88, 2000.

P. Milgram and F. Kishino. A taxonomy of mixed reality visual displays. IEICE Transactions on Information Systems, E77-D (12): 1321-1329, 1994.

P. Milgram and S. Zhai. Applications of augmented reality for human-robot communications. Proceedings of 1993 IEEE/RSJ International Conference on Intelligent Robots and Systems, 1467- 1476, 1993.

Y. Mukaigawa, S. Mihashi, and T. Shakunaga. Photometric imagebased rendering for virtual lighting image synthesis. Proc. 2nd Int.l Workshop Augmented Reality (IWAR '99), 115-124, 1999.

Ryutarou Ohbuchi, David Chen, and Henry Fuchs. Incremental volume reconstruction and rendering for 3D ultrasound imaging. Visualization in Biomedical Computing, 1808: 312-323, 1992.

H.L. Pryor, T.A. Furness, and E. Viirre. The virtual retinal display: A new display technology using scanned laser light. Proc. 42nd Human Factors Ergonomics Society, pp. 1149, 1998.

F. Sauer, A. Khamene, B. Bascle, L. Schimmang, F. Wenzel, and S. Vogt. Augmented reality visualization of ultrasound images: System description, calibration and features. IEEE and ACM International Symposium on Augmented Reality, 30-39, 2001.

T.B. Sheridan. Musing on telepresence and virtual presence. Presence, 1(1):120-125, 1992.

W. Sherman and A. Craig. Understanding Virtual Reality: Interface, Applications and Design. Morgan Kaufmann Publishers, 2003.

R. Silva and G. Giraldi. Introduction to virtual reality. Technical Report: 06/2003, LNCC, Brazil, 2003.

D. Sims. New realities in aircraft design and manufacture. IEEE Computer Graphics and Applications, 14 (2): 91 , 1994.

J. Stauder. Augmented reality with automatic illumination control incorporating ellipsoidal models. IEEE Trans. Multimedia, 1 (2): 136-143, 1999.

Z. Szalavri, E. Eckstein, and M. Gervautz. Collaborative gaming in augmented reality. VRST, Taipei, Taiwan, 195204, 1998.

E. Urban. The information warrior. IEEE Spectrum, 32 (11): 66-70, 1995.

D. Weimer. Frontiers of Scientific Visualization, chapter 9,"Brave New VirtualWorlds". Pages 245-278. John Wiley and Sons, Inc., 1994.11 